\title{
LA GRAMÁTICA UNIVERSAL EN EL PROGRAMA MINIMISTA*
}

\section{UNIVERSAL GRAMMAR IN THE MINIMALIST PROGRAM}

\section{LUIS EGUREN}

Universidad Autónoma de Madrid. Madrid, España

luis.eguren@uam.es

\section{RESUMEN}

En este artículo se estudian distintos aspectos de una de las propuestas más innovadoras del Programa Minimista (PM) chomskiano en comparación con etapas anteriores de la Gramática Generativa: la idea de que la Gramática Universal (GU) está infraespecificada. Se identifica, en concreto, el contenido que se atribuye en el PM a la GU, se exponen las causas que motivan su reducción y se analizan las implicaciones que la infradeterminación minimista de la GU tiene tanto para la debatida cuestión de la especificidad del lenguaje humano como para los estudios sobre la adquisición del lenguaje.

Palabras clave: Programa Minimista, Gramática Universal, ensamble, tercer factor.

\section{ABSTRACT}

This paper addresses various aspects of a novel proposal made by the chomskyan Minimalist Program (MP), as compared with previous stages in Generative Grammar: the idea of an underspecified Universal Grammar (UG). The content attributed to UG within the MP is identified, the reasons underlying the minimalist reduction of UG are made explicit, and the effects of the underdetermination of UG are analyzed with respect to both the issue of the specificity of human language and the studies on first language acquisition.

Keywords: Minimalist Program, Universal Grammar, merge, third factor.

Recibido: 15/01/2013. Aceptado: 19/08/2013.

${ }^{*}$ Este trabajo ha sido parcialmente financiado gracias a la ayuda concedida al proyecto FFI201123829. 


\section{INTRODUCCIÓN}

Oomo han señalado distintos autores ${ }^{1}$, podría muy bien afirmarse que la lingüística chomskiana constituye un programa de investigación, en el sentido que el filósofo de la ciencia Imre Lakatos da a este término, organizado en torno a un "centro firme" invariable: la adopción de un enfoque biolingüístico según el cual las lenguas son estados de la mente de los individuos, "órganos mentales", cuyo estudio debe ser abordado haciendo uso de los mismos criterios y procedimientos con que las ciencias naturales estudian los objetos del mundo. Internismo y naturalismo metodológico son, pues, dos componentes nucleares del programa de investigación chomskiano, a los que hay que añadir un tercero: la concepción computacional del sistema cognitivo lingüístico, la idea de que el conocimiento lingüístico de los individuos consiste en algoritmos u operaciones que manipulan "representaciones" mentales formadas por unidades simbólicas portadoras de información.

El centro firme de la lingüística chomskiana se ha mantenido intacto desde hace ya más de cinco décadas, pero también se han producido cambios sustanciales, que en última instancia se explican en función de las distintas preguntas a las que Noam Chomsky ha intentado dar respuesta en cada una de las tres etapas de su teoría: a) ¿qué es el conocimiento lingüístico? (en la Teoría Estándar), b) ¿cómo se adquiere el lenguaje? (en la Teoría de los Principios y los Parámetros), c) ¿por qué las propiedades del lenguaje son las que son? (en el actual Programa Minimista) (cf. Chomsky, 2004a, 2004b).

Uno de estos cambios ha afectado, en concreto, al contenido atribuido a la dotación innata para el lenguaje específica de la especie humana, la llamada "Gramática Universal" $(\mathrm{GU})^{2}$ : sorprendentemente para algunos, y en claro contraste con lo postulado por la Teoría de los Principios y los Parámetros, el Programa Minimista (PM) aboga por una drástica reducción de la GU, que incluiría ahora, en esencia, un conjunto de propiedades o rasgos (fonéticos, formales y semánticos) y una sola operación computacional de ensamble (Merge), que agrupa las unidades léxicas formadas con tales rasgos y genera de este modo unidades sintagmáticas estructuradas.

En las páginas que siguen se analizará con detalle algunos aspectos de este importante giro en el pensamiento lingüístico chomskiano. Se identificará en par-

${ }^{1}$ Véase Eguren y Fernández Soriano (2004) y las referencias allí citadas.

${ }^{2}$ Como señala acertadamente Laka (2009), innatismo y especificidad no son sinónimos, dado que un rasgo puede ser innato en una especie sin ser específico de dicha especie. De la definición de la GU presentada en el texto se deduce, por tanto, que para atribuir una propiedad a la GU no basta con que sea innata, sino que ha de ser también específica tanto del sistema cognitivo lingüístico, frente a otros dominios cognitivos, como de la especie humana, en comparación con los sistemas de comunicación de otras especies (véase el apartado 4). 
ticular, primero, las razones que motivan la infraespecificación de la GU en el Programa Minimista. Se abordará después la cuestión de la especificidad del lenguaje humano, que el PM nos hace reconsiderar con una nueva óptica, centrándome en la operación de ensamble. Y se tratará de precisar, finalmente, qué implicaciones tiene la idea minimista de una GU infraespecificada para los estudios sobre la adquisición del lenguaje. Pero antes de entrar en materia, y para que el lector pueda valorar cabalmente el sentido y el alcance de las propuestas minimistas, en el siguiente apartado se detallará brevemente cuál es el contenido con que se dota a la GU en la Teoría Estándar y en la Teoría de los Principios y los Parámetros.

\section{DE LAS REGLAS A LOS PRINCIPIOS Y LOS PARÁMETROS}

La Teoría Estándar (Chomsky, 1965) tiene como objetivo describir las propiedades de las gramáticas mentales de los hablantes de manera explícita y exhaustiva. Con el propósito de cumplir el requisito de "adecuación descriptiva" de la teoría, en esta fase inicial de la lingüística chomskiana se construye un complejo sistema de reglas de estructura de frase, que expresan la estructura de constituyentes de los sintagmas y las oraciones (1), y de reglas transformacionales, con las que se obtienen representaciones derivadas a partir de formas subyacentes y se capta así el hecho, por ejemplo, de que en una oración interrogativa parcial como ¿Qué leyó Juan? el pronombre interrogativo qué se pronuncia al principio de la oración, pero se interpreta como el objeto directo de leer (2):

1) Juan leyó el libro.
a. $\mathrm{O} \rightarrow \mathrm{SN}+\mathrm{SV}$
b. $\mathrm{SV} \rightarrow \mathrm{V}+\mathrm{SN}$

2) Juan leyó qué $\rightarrow \quad$ ¿Qué leyó Juan?

(Estructura profunda) (Estructura superficial)

En consonancia con la caracterización del conocimiento gramatical como un sistema de reglas, en Aspectos de la teoría de la sintaxis se propone que la Gramática Universal especifica cuáles son los dos tipos permitidos de reglas (de estructura de frase y transformacionales) y la manera en que éstas se aplican. Además de estos "universales formales", considera Chomsky en Aspectos de la teoría de la sintaxis (1965) que la GU proporciona también un conjunto de "universales sustantivos" (un listado de rasgos fonéticos, sintácticos y semánticos comunes a todas las lenguas), así como una "medida de evaluación", que permite escoger entre las gramáticas que sean extensionalmente equivalentes aquellas que capten generalizaciones significativas empleando las notaciones más simples. La GU tiene, por tanto, un 
contenido muy reducido en la Teoría Estándar, pero no porque fuera esto lo que se pretendía, como luego ocurrirá en el Programa Minimista, sino debido a que, en palabras del propio Chomsky (1965: 44), en esta primera etapa se estaba aún "muy lejos de poder presentar un sistema de universales lingüísticos formales y sustantivos que sea lo suficientemente rico y detallado para dar razón de los hechos del aprendizaje del lenguaje".

El modelo de los Principios y los Parámetros (o Teoría de la Rección y el Ligamiento) desarrollado en la década de los ochenta y en los primeros años noventa del siglo pasado (cf., v.g., Chomsky, 1981, 1986; Chomsky y Lasnik, 1993) persigue un nuevo objetivo, intenta diseñar una teoría gramatical explicativamente adecuada, que dé cuenta de cómo adquieren los niños su lengua materna y nos permita solucionar, en concreto, el denominado "problema lógico de la adquisición del lenguaje": ¿ cómo es posible que el niño que aprende una lengua desarrolle en su mente un conocimiento lingüístico extremadamente rico y articulado a partir de una experiencia lingüística imperfecta, limitada e, incluso a veces, inexistente?

Un paso previo que la Teoría P\&P da para tratar de alcanzar el requisito de "adecuación explicativa" de la teoría es concebir el conocimiento gramatical de los hablantes no como un sistema de reglas, como se hacía en Aspectos de la teoría de la sintaxis (1965), sino como la interacción de las propiedades del léxico que se proyectan en la sintaxis con principios de buena formación de las derivaciones y las representaciones. La razón de este cambio reside en que, mientras que las piezas léxicas de una lengua han de ser en todo caso aprendidas, no parece plausible que aprender la lengua materna, en las condiciones en que ello se produce (sin instrucción aparente, en un corto período de tiempo y de manera uniforme para todos los miembros de la especie), pueda consistir en adquirir reglas que a menudo son exclusivas de cada lengua e incluyen una información idiosincrásica, heterogénea o redundante.

Una vez sustituidos los sistemas de reglas por la combinación de rasgos léxicos sintácticamente activos y principios de buena formación como modo de caracterizar el conocimiento gramatical, la Teoría P\&P intenta resolver la versión lingüística del "problema de Platón" atribuyendo un rico contenido a la GU. La idea es que se puede reducir la distancia entre la pobreza de los estímulos lingüísticos que el niño percibe en su entorno y la riqueza del conocimiento lingüístico que "crece" en su mente si suponemos que dicho conocimiento está en buena medida predeterminado de manera innata.

La Teoría P\&P propugna, a este respecto, que la GU contiene, en primer lugar, un buen número de principios que restringen las propiedades de las gramáticas, como, por ejemplo, los axiomas de la Teoría de la X con barra, que regulan la buena formación de las estructuras de frase, las condiciones de la Teoría Temática, que afectan a las estructuras argumentales de los predicados, el Filtro del Caso, con el que se da cuenta de los contextos sintácticos en que aparecen las expresio- 
nes nominales, el Principio de Proyección Ampliado, que establece que todas las oraciones deben tener un sujeto, o el Principio de las Categorías Vacías, que limita los "movimientos" de unidades léxicas y sintagmas desde las posiciones en que se interpretan semánticamente a las posiciones en que se pronuncian.

Junto con principios invariables como los mencionados, para explicar la diversidad lingüística, la Teoría P\&P sostiene que forma igualmente parte de la GU una serie de parámetros de variación sintáctica, u opciones abiertas entre valores determinados de manera innata cuya fijación puede tener repercusiones sistemáticas en otros aspectos de la gramática de una lengua, como, entre otros, el Parámetro de la Posición del Núcleo, que recoge el hecho de que los núcleos preceden a sus complementos en unas lenguas y los siguen en otras, el Parámetro del Sujeto Nulo, que divide las lenguas entre las que admiten sujetos elididos y las que los rechazan, o el Parámetro del Movimiento $\mathrm{Cu}$, que distingue entre las lenguas en que los pronombres y adverbios interrogativos se sitúan al principio de la oración y aquellas en las que permanecen in situ. Pero no es esto todo. En esta segunda etapa de la lingüística chomskiana, la GU contiene además, como en Aspectos de la teoría de la sintaxis (1965), un inventario de rasgos o propiedades universales, aporta también una operación ("muévase $\alpha$ "), que reemplaza a las transformaciones de movimiento de la Teoría Estándar, y especifica incluso cuál es la arquitectura del sistema gramatical (cf. Chomsky, 1981).

En la Teoría de los Principios y los Parámetros, en conclusión, la Gramática Universal está claramente sobredimensionada. El panorama será muy distinto en el Programa Minimista. Como se verá a continuación, la adopción de una perspectiva minimista sobre las propiedades de la facultad del lenguaje traerá necesariamente consigo la infraespecificación del contenido de la GU.

\section{LA INFRADETERMINACIÓN MINIMISTA DE LA GRAMÁTICA UNIVERSAL}

El Programa Minimista coincide con la Teoría de los Principios y los Parámetros en la caracterización del conocimiento gramatical de los hablantes como la combinación de rasgos, operaciones y principios, y no como un conjunto de reglas, como se proponía en Aspectos de la teoría de la sintaxis (1965). Es en este sentido en el que cabría decir que el Programa Minimista es una extensión de la Teoría P\&P (cf. Eguren y Fernández Soriano, 2004). Existen, sin embargo, profundas diferencias entre el Minimismo y la Teoría P\&P que se derivan, como apuntaba en la introducción, del hecho de que, en las dos últimas décadas, la lingüística de inspiración chomskiana haya intentado dar respuesta a una pregunta de nuevo cuño y gran alcance: ¿por qué la facultad del lenguaje tiene precisamente las propiedades que tiene? El PM no se contenta, por tanto, con solucionar el problema lógico de 
la adquisición del lenguaje, sino que quiere ir "más allá de la adecuación explicativa”, y pretende ofrecer una explicación fundamentada, basada en principios, de al menos una parte sustancial de las propiedades del sistema cognitivo lingüístico. La propuesta del PM a este respecto es que los principios que explican por qué determinadas propiedades de la facultad del lenguaje son las que pertenecen, en concreto, al denominado "tercer factor", en el que se incluyen condiciones no específicas de la facultad del lenguaje (e independientes incluso del organismo) que intervienen, junto con la dotación genética (la GU) y la experiencia lingüística, en la adquisición del lenguaje (cf. Chomsky, 2004a, 2004b, 2005, 2007, 2008, 2010; Berwick y Chomsky, 2011).

La idea minimista de que ciertos principios no específicos del lenguaje desempeñan un papel determinante en su diseño y adquisición surge -no se debe pasar esto por alto- del "centro firme" de la lingüística chomskiana, el enfoque biolingüístico del lenguaje y las lenguas: Si se asume que la facultad del lenguaje tiene las mismas propiedades que otros sistemas orgánicos, se puede perfectamente establecer un paralelismo entre los factores que se cree que entran en juego en el desarrollo y la evolución de los organismos y los implicados en el "crecimiento" del "órgano del lenguaje" en los individuos. Los tres componentes del modelo minimista de adquisición del lenguaje (la GU, los estímulos lingüísticos del entorno y las condiciones externas) se corresponderían, así, con los tres factores que Jacques Monod, en Le hasard et la nécessité (1970) o Stephen Gould, en The structure of evolutionary theory (2002), identifican en la evolución de las especies: a) la información genética (canales del desarrollo predeterminados, mutaciones al azar), b) la selección natural (la adaptación a las presiones ambientales) y c) restricciones estructurales, leyes generales de la física o de la química que limitan la variedad y la forma de los organismos y los sistemas complejos, como el hecho, por poner un ejemplo ilustrativo, de que sea casi imposible emplear en el mundo orgánico ruedas para la locomoción debido a las dificultades físicas que entraña proporcionar control nervioso y suministro de sangre a un objeto rotatorio (cf. Berwick y Chomsky, 2011: 22)3 . Noam Chomsky aplica, por tanto, el modelo tripartito de la evolución propuesto por la biología moderna al estudio de las propiedades de la facultad del lenguaje.

En lo concerniente a esta facultad de la mente, sostiene Chomsky (2005: 6) que "el tercer factor se divide en distintos subtipos: a) principios de análisis de los datos que podrían ser empleados en la adquisición del lenguaje y en otros dominios; b) principios de arquitectura estructural y restricciones sobre el desarrollo...,

\footnotetext{
${ }^{3}$ El lector puede encontrar referencias más detalladas a los autores que, como Monod o Gould, sostienen que existe un "tercer factor" en la evolución y el desarrollo de los organismos, así como indicaciones sobre los distintos tipos de restricciones que se atribuyen a dicho factor, en Berwick y Chomsky (2011: 22-25), Boeckx (2009: 46), Chomsky (2004a: 104-105, 2005: 5-6, 2010: 50) y Longa, Lorenzo y Uriagereka (2011: 598-99).
} 
incluidos los principios de computación eficiente, que debería esperarse que tuvieran especial relevancia para sistemas computacionales como el lenguaje" ${ }^{4}$. Y añade que "es la segunda de estas subcategorías la que debería ser especialmente relevante a la hora de determinar la naturaleza de las lenguas que se pueden adquirir”. El Programa Minimista considera, así, que son dos en particular los tipos de principios del tercer factor que pueden ayudarnos a explicar las propiedades de la facultad del lenguaje: principios, por un lado, de arquitectura estructural y condiciones de economía o eficiencia computacional, por otro. Veamos en qué consisten dichos principios, empezando por los principios de arquitectura estructural.

Una constante en el pensamiento lingüístico chomskiano es su concepción de la arquitectura de la facultad del lenguaje. Ya en la Teoría P\&P, y con mayor énfasis en el PM, sostiene Chomsky que la facultad del lenguaje está constituida por tres sistemas diferenciados: un sistema cognitivo lingüístico (o "lengua-I"), formado por un léxico y por mecanismos computacionales de uno u otro tipo, y dos sistemas de actuación, el sistema sensorio-motriz y el sistema conceptual-intencional, que utilizan las "expresiones" generadas por las gramáticas mentales para la articulación y la percepción, la formación de conceptos, para referirse al mundo, etc. Los dos sistemas de actuación son, como el sistema cognitivo lingüístico, internos al organismo, pero, al contrario de éste, que se supone que está especializado para el lenguaje, poseen propiedades y desempeñan funciones que son independientes de la facultad del lenguaje: el sistema sensorio-motriz nos sirve también para articular o percibir sonidos no lingüísticos, y parece igualmente posible tener pensamientos sin lenguaje. Estos tres sistemas de la mente están interrelacionados, de modo que el sistema cognitivo lingüístico sirve de puente entre los dos sistemas de actuación y debe entrar necesariamente en contacto con ellos para poder ser usado. Pues bien, dando por sentado que la facultad del lenguaje contiene estos tres sistemas y que dichos sistemas se conectan entre sí tal y como se ha expuesto, los principios de arquitectura estructural del tercer factor son principios explicativos relacionados con las "condiciones de interficie" impuestas sobre el sistema cognitivo lingüístico por los sistemas sensorio-motriz y conceptual-intencional con los que interacciona. Así, por ejemplo, una propiedad definitoria del lenguaje oral como es la linearización se explicaría de manera externa como un requisito del sistema sensorio-motriz, que impone, dada la naturaleza del aparato articulatorio, que las unidades lingüísticas deban sucederse en el tiempo. De igual modo, la existencia en todas las lenguas del mundo de estructuras predicado-argumentos o de relaciones operador-variable sería una exigencia del sistema del pensamiento que, como hemos dicho, es, al menos en parte, independiente del lenguajes.

${ }^{4}$ Todas las traducciones del inglés en el texto y en las notas son del autor.

${ }^{5} \mathrm{La}$ idea de que las condiciones de interficie pertenecen al tercer factor (constituyen principios de arquitectura estructural extralingüística) se expresa con claridad en Chomsky (2005: 9-10). Paradójicamente, el propio Chomsky parece, en ocasiones, sugerir que este tipo de principios está 
El segundo tipo de principios del tercer factor que, según Noam Chomsky, explican por qué la facultad del lenguaje tiene las propiedades que tiene son las condiciones de computación eficiente que el sistema cognitivo lingüístico debe respetar en tanto que sistema computacional, unas condiciones que afectan a cualquier sistema de esta índole y que son, por tanto, no solo extralingüísticas, como los principios de arquitectura estructural, sino también externas al organismo. Por mencionar tan solo algunas de ellas, se trata de condiciones como la prohibición de que haya elementos superfluos en las representaciones (el llamado "Principio de Interpretación Plena") o pasos inmotivados en las derivaciones, la exigencia de que los movimientos sean lo más cortos posibles, la determinación local de las operaciones, evitando mirar hacia adelante (look-ahead) y hacia atrás (backtracking) en una derivación o comparar alternativas de modo global, la restricción de que las operaciones se limiten a reajustar objetos sintácticos ya generados en el curso de la derivación, sin insertar símbolos nuevos (no tampering), etc. (véase, v.g., Chomsky, 2000: 99; 2004b: xii).

A modo de inciso, quisiera hacer notar que, al escoger los dos tipos de principios explicativos mencionados, la lingüística chomskiana se reafirma en su "centro firme", profundiza en la concepción internista y computacionalista del lenguaje que, como señalaba en la introducción, ha mantenido a lo largo de toda su historia: Solo partiendo de la premisa de que las lenguas son estados de la mente de naturaleza computacional se puede buscar la explicación de sus propiedades en la interacción del sistema cognitivo lingüístico con otros sistemas mentales o en características propias de los sistemas computacionales.

Tras identificar las clases de principios del tercer factor que podrían explicar las propiedades de la facultad del lenguaje, el PM pone en marcha un ambicioso programa de investigación inspirado por lo que se conoce como "Tesis Minimista Fuerte", la idea de que la facultad del lenguaje es una solución "óptima" (sujeta a condiciones de eficiencia computacional) para las condiciones de interficie impuestas por el sistema sensorio-motriz y el sistema conceptual-intencional. Las investigaciones minimistas someterán a prueba esta idea, se empeñarán en comprobar hasta qué punto la Tesis Minimista Fuerte es cierta, tratarán de determinar, en fin, qué propiedades de la facultad del lenguaje encuentran su explicación en condiciones del tercer factor y cuáles no.

Hasta aquí han quedado expuestos los fundamentos y los objetivos del Programa Minimista. Volvamos ahora al tema central de este trabajo. La Tesis Minimista Fuerte tiene un impacto directo sobre el contenido que en el PM se asigna a la Gramática Universal. La búsqueda minimista de una explicación de las propiedades del lenguaje a partir de principios del tercer factor trae como consecuencia que se intente reducir al máximo el contenido de la GU. La razón es que atribuir una

codificado en la GU (cf. Chomsky, 2004a: 106; 2008: 134). 
determinada propiedad a la GU (la dotación genética para el lenguaje) equivale a renunciar a explicarla de manera independiente, en los términos de la Tesis Minimista Fuerte (que haya rasgos del lenguaje que pertenecen a la GU se justificaría de otro modo, recurriendo quizás a procesos evolutivos o propiedades del cerebro que nos son desconocidos). En el PM se descompone, en consecuencia, el contenido que la GU tenía en la Teoría P\&P y se extraen de este primer factor cuantos elementos puedan explicarse por medio de principios del tercer factor.

En un primer momento, los principios que la Teoría P\&P ubicaba en la GU se reformulan en el PM como condiciones de interficie o de eficiencia computacional (cf. Chomsky, 1995, 2000, 2001). Así, por ejemplo, el Criterio Temático, que requiere, como se ilustra parcialmente en el paradigma de (3), que todo papel temático (o función semántica) haya de ser asignado a un (solo) argumento y que todo argumento deba tener un (solo) papel temático, se concibe ahora como una condición de la interficie semántica impuesta por el sistema conceptual-intencional:

3) a. Juan lanzó la piedra ('lanzar' = <agente, tema $>$ )

b. *Juan lanzó.

c. *Juan lanzó la piedra la moneda.

Igualmente, por mencionar tan solo otro caso, los efectos de la llamada "Minimidad Relativizada”, que en la Teoría P\&P se vinculaban a principios de la GU, se explican en el PM como el resultado de una condición de eficiencia computacional, la Condición del Movimiento Más Corto. Así, la agramaticalidad de una oración como la de (4b) no se atribuye ya a una infracción del Principio de las Categorías Vacías, producida porque la presencia del sintagma interrogativo qué casa desplazado al principio de la oración subordinada actúa como una "barrera" para que el adverbio interrogativo cuándo que introduce la oración principal pueda regir propiamente a su huella. La oración de (4b) estaría mal formada debido a que se infringe una condición de economía computacional, según la cual, en el caso de que haya más de un elemento que pueda moverse a una determinada posición, el que debe desplazarse es el elemento que esté más próximo estructuralmente a dicha posición (en nuestro ejemplo, qué casa, y no el adverbio cuándo).

4) a. ¿Cuándo te dijo Juan [ $\mathrm{h}_{\mathrm{i}}$ que compraría la casa Pedro $\mathrm{h}_{\mathrm{i}}$ ]?

b. ${ }_{i}^{*} C u a ́ n d o_{\mathrm{i}}$ te dijo Juan [ qué casa ${ }_{\mathrm{j}}$ compraría $\mathrm{h}_{\mathrm{j}}$ Pedro $\mathrm{h}_{\mathrm{i}}$ ] ?

Más recientemente se defiende la idea, en un número creciente de investigaciones minimistas, de que los parámetros de variación sintáctica tampoco forman parte de la GU, sino que son propiedades emergentes de los sistemas lingüísticos, cuyo origen y alcance dependen de condiciones externas al lenguaje (cf., v.g., Ro- 
berts y Holmberg, 2005, 2010; Holmberg, 2010; Boeckx, 2011). Un caso paradigmático del modo en que se intenta dar cuenta de la variación paramétrica en las propuestas actuales del PM es el del Parámetro de la Posición del Núcleo, que divide las lenguas, como sabemos, en dos grandes tipos: lenguas en que los núcleos se sitúan delante de sus complementos, como el español (5), y lenguas en que se colocan detrás, como el vasco (6).

5) a. Lanzó la piedra.

b. Sin cabeza.

6)
a. Harria bota zuen. $(\mathrm{SN}+\mathrm{V})$ piedra.la lanzar aux
b. Bururik cabeza.part
$(\mathrm{SN}+\mathrm{P})$

$(\mathrm{P}+\mathrm{SN})$
$(\mathrm{V}+\mathrm{SN})$ (español)

(vasco)

Para la Teoría P\&P, este parámetro, con sus dos opciones (los núcleos preceden o siguen a sus complementos) pertenece a la GU. En el PM, en cambio, el que existan justo estas dos posibilidades no estaría predeterminado por la GU, sino que se deriva de una propiedad física del tercer factor (no específica de la facultad del lenguaje): la naturaleza de nuestro aparato articulatorio hace que el núcleo de un sintagma y su complemento, como cualquier otra unidad lingüística, se deban pronunciar uno tras otro, dando lugar a tan solo dos opciones (Holmberg, 2010). El constatado hecho de que los núcleos tienden a preceder o seguir a sus complementos de manera uniforme en una lengua determinada sería, a su vez, consecuencia de la aplicación, en el proceso de adquisición de las lenguas maternas, de mecanismos generales de aprendizaje no exclusivos del lenguaje, unos mecanismos pertenecientes de nuevo, por tanto, al tercer factor, como la "generalización del input" (Roberts y Holmberg, 2010) o el "sesgo del superconjunto" (Boeckx, 2011), que favorecen la consolidación de sistemas armónicos u homogéneos.

Como acabo de ilustrar, la causa principal de la reducción del contenido de la GU en el PM se encuentra, en suma, en el objetivo mismo que el actual desarrollo de la lingüística chomskiana persigue: explicar, en la medida de lo posible, las propiedades de la facultad del lenguaje por medio de principios del tercer factor. En sus escritos de los últimos años, Chomsky añade a este otro motivo para que la GU deba tener un contenido reducido: solo con un GU infradeterminada se puede ofrecer una explicación plausible de la evolución del lenguaje (cf. Chomsky, 2005, 2007, 2008). Veamos en qué se basa esta afirmación.

$\mathrm{Al}$ igual que otros estudiosos de este enigmático fenómeno, Noam Chomsky tiene una visión rupturista de la evolución del lenguaje, según la cual, a grandes rasgos, las propiedades específicas del lenguaje humano aparecieron hace unos 50.000 ó 100.000 años, posiblemente como resultado de una mutación genéti- 
ca que produjo cambios en el cerebro de una pequeña población en el linaje de los homínidos (cf. Chomsky, 2010: 58-59; Berwick y Chomsky, 2011: 26-27, 40-41). Si se acepta esta concepción discontinuista de la evolución del lenguaje, el asunto está entonces en afrontar lo que Cedric Boeckx (2009: 45) llama el "problema de Darwin": ¿Cómo surgieron hace tan poco tiempo, y de manera tan abrupta, las propiedades cualitativamente distintas que caracterizan al lenguaje humano? En opinión de Chomsky, la solución a este problema pasa por asignar el menor contenido posible a la GU, dado que "cuanto menor sea el peso de la información genética (en este caso, la GU) a la hora de determinar el desarrollo de un organismo, más factible es el estudio de su evolución” (Chomsky, 2007: 4) o, dicho con otras palabras, "cuanto más variadas y complejas sean las condiciones específicas del lenguaje, menos esperanzas tendremos de poder dar cuenta de los orígenes evolutivos de la GU" (Chomsky, 2005: 8).

¿Cuál es entonces para el Programa Minimista, querrá saber el lector, el contenido de una GU infraespecificada de la que se han extraído, por las razones apuntadas, los principios y los parámetros? Como se deduce de lo dicho hasta ahora, la respuesta minimista a esta cuestión solo puede ser ésta: la GU consiste únicamente en aquellas propiedades que no se puedan explicar recurriendo a principios del tercer factor y que tuvieron que surgir de algún modo, por tanto, en el transcurso de la evolución del lenguaje (cf. Chomsky, 2007: 5). En sus trabajos de las dos últimas décadas, Noam Chomsky siempre ha propuesto a este respecto que una GU minimizada tiene, básicamente, dos componentes: un conjunto de rasgos (o propiedades lingüísticas) con el que se forman unidades léxicas y un mecanismo computacional, la operación de ensamble, que combina las piezas léxicas para construir expresiones lingüísticas complejas (cf., v.g., Chomsky, 2000: 100; 2004a: 107-108; 2007: 5-6) . El siguiente párrafo ilustra su propuesta:

...surgen al menos dos problemas fundamentales cuando analizamos los orígenes de la facultad del lenguaje y su papel en la repentina aparición de las capacidades intelectivas humanas: en primer lugar, las propiedades semánticas básicas de las unidades mínimas con significado...; y, en segundo lugar, los principios que permiten la combinación ilimitada de símbolos jerárquicamente organizados, con la que se posibilita el uso del lenguaje en sus diversas funciones. En consecuencia, la teoría de las propiedades nucleares del lenguaje (la Gramática Universal) debe

\footnotetext{
${ }^{6}$ Chomsky (2000: 100) considera que, además del ensamble, pertenece también a la GU la relación de concordancia (Agree) entre una sonda (probe) y una meta (goal) que borra de las expresiones lingüísticas los rasgos no interpretables para el sistema conceptual-intencional. Chomsky (2004a) piensa, en cambio, que esta relación podría derivarse de propiedades del tercer factor si suponemos que el borrado de rasgos no interpretables es el mecanismo que activa el "desplazamiento" (véase más abajo en el texto) y que la aplicación de dicho mecanismo responde a determinadas condiciones de la interficie semántica.
} 
proporcionar, primero, un inventario estructurado de unidades léxicas posibles..., y segundo, mecanismos para construir a partir de estas unidades léxicas la infinita variedad de estructuras internas que participan en el pensamiento, la interpretación, la planificación y otras acciones de la mente humana... (Chomsky, 2005: 4).

La operación de ensamble, en concreto, aplicada de manera recurrente e ilimitada nos permite captar una de las propiedades más definitorias del lenguaje humano: la infinitud discreta, la capacidad de producir un número potencialmente infinito de expresiones jerárquicamente estructuradas a partir de un número finito de unidades ${ }^{7}$. Se distinguen dos tipos de ensamble, ambos pertenecientes a la GU: el ensamble externo, con el que se forman las estructuras de constituyentes, y el ensamble interno, que da cuenta, además, de la "propiedad del desplazamiento", el hecho de que las unidades lingüísticas a menudo se interpretan semánticamente en una posición y se pronuncian en otra (cf., v.g., Chomsky, 2004a: 10; 2005: 12; 2008: 140; 2010: 54). El ensamble externo toma dos "objetos lingüísticos" diferenciados $\mathrm{X}$ e $\mathrm{Y}$ y construye con ellos un nuevo objeto lingüístico $\mathrm{Z}$ que tiene las propiedades bien de $\mathrm{X}$, bien de $\mathrm{Y}(7 \mathrm{a})$ : reúne, por poner un ejemplo sencillo, las palabras leer y libros y forma con ellas un conjunto con propiedades verbales (7b).

7)

a.

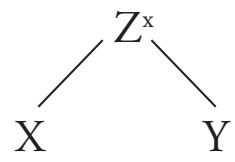

b.

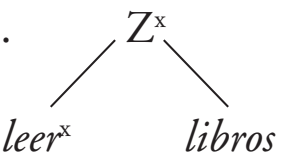

El ensamble interno, que desempeña el papel de las transformaciones de movimiento de la Teoría Estándar o de la operación "muévase $\alpha$ " de la Teoría P\&P, toma, a su vez, un objeto $\mathrm{X}$ y un objeto $\mathrm{Y}$ que está incluido en $X \mathrm{y}$ ensambla $\mathrm{Y}$ con $\mathrm{X}$, formando un objeto con los rasgos de X (8a). Para captar, por ejemplo, que en una oración como ¿Qué leyó? el pronombre interrogativo se pronuncia al principio de la oración pero se interpreta como el OD de leer, esta operación, como se ve en $(8 \mathrm{~b})$, parte del conjunto ya formado leyo qué y crea una copia de un miembro de ese conjunto (el interrogativo qué) que se ensambla con el conjunto: ¿qué leyó

${ }^{7}$ Chomsky $(2007,2008)$ sugiere que la naturaleza ilimitada del ensamble se podría expresar por medio de un "rasgo de margen" (edge feature) que permite que una pieza léxica se ensamble con otra. Dicho rasgo permanecía siempre activo, y haría así posible que los objetos sintácticos se ensamblen "en los márgenes" de objetos ya formados hasta el infinito. Concluye, por tanto, Chomsky (2007: 11) que "...las únicas propiedades sintácticas de la GU son el ensamble y unidades léxicas con un rasgo de margen imborrable...”. A esto añade después que también es una propiedad de la GU "el que las expresiones generadas deban satisfacer condiciones de interficie", y apunta en otro lugar que "además de estas propiedades, la GU debe contener los principios que convierten los datos externos en experiencia lingüística, proporcionando las bases para la adquisición del lenguaje" (Chomsky, 2007: 7). Estas citas ilustran que Chomsky parece tener en mente que el contenido de la GU no solo consiste en un conjunto de rasgos léxicos y en la operación de ensamble. 
qué? En la interficie semántica se interpretan ambas copias, la más alta como un operador y la más baja como una variable ('para qué x, leyó x'), pero en la interficie sensorio-motriz solo se pronuncia la primera, y la segunda se borra (¿qué leyó?).

8)

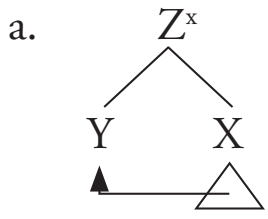

b.

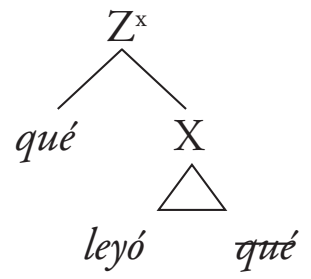

En conclusión, la GU está formada esencialmente, para el Programa Minimista chomskiano, por un inventario de rasgos léxicos y por una única operación computacional de ensamble. Nótese, sin embargo, que la Tesis Minimista Fuerte (la idea de que la facultad del lenguaje es una solución computacionalmente eficiente para las condiciones de interficie), de ser totalmente cierta, implicaría que la GU no tiene contenido alguno, dado que todas las propiedades del lenguaje se explicarían de manera externa (cf. Chomsky, 2004a: 106). El PM pone de nuevo sobre el tapete, por tanto, la cuestión de la especificidad del lenguaje humano, aunque desde una nueva perspectiva, y nos fuerza a comprobar si propiedades incluso como la operación de ensamble son realmente exclusivas del sistema cognitivo lingüístico. Del actual debate en torno a este asunto trata el siguiente apartado ${ }^{8}$.

\section{LA ESPECIFICIDAD DEL LENGUAJE HUMANO}

Con la intención de centrar y esclarecer la discusión sobre la unicidad del lenguaje humano, Noam Chomsky, en dos influyentes artículos escritos en colaboración con Marc D. Hauser y W. Tecumseh Fitch, traza una distinción entre dos concepciones distintas de la facultad del lenguaje, una más general y abarcadora, "la facultad del lenguaje en sentido amplio", y otra más restringida y excluyente, "la facultad del lenguaje en sentido estricto" (cf. Hauser, Chomsky y Fitch, 2002; Fitch, Hauser y Chomsky, 2005). La facultad del lenguaje en sentido amplio (FLA) contiene, en opinión de estos autores, a la facultad del lenguaje en sentido estricto

\footnotetext{
${ }^{8}$ Señala un revisor anónimo que "en su núcleo, la idea de que la GU, en los términos tradicionalmente expuestos por los generativistas, no existiría o sería mínima, y que, consecuentemente, tanto filogenética como ontogenéticamente el lenguaje respondería, más bien, a procesos de corte emergentista, ha venido desarrollándose en la bibliografía especializada desde la década de 1990" y sugiere que "los enfoques chomskianos actuales deberían evaluarse sobre el trasfondo de esta discusión más amplia respecto de la naturaleza y el origen del lenguaje". Se coincide con dicha observación. No obstante, una comparación detallada y crítica de la concepción minimista de la GU y de las teorías emergentistas sobrepasa con creces los límites y el objetivo de este trabajo y queda pendiente de futuras investigaciones.
} 
(FLE) e incluye además, al menos, los mecanismos de los sistemas de actuación conceptual-intencional y sensorio-motriz internos a la mente implicados en el pensamiento y el uso del lenguaje, unos mecanismos que en su mayor parte (quizás en su totalidad) se encuentran en otros dominios cognitivos y de los que hacen uso otras especies, con diferencias de grado, no cualitativas. La FLA engloba, por tanto, todas las capacidades que participan en el lenguaje, con independencia de que sean específicas de este dominio cognitivo o exclusivamente humanas. La facultad del lenguaje en sentido estricto (FLE) es un subcomponente de la FLA y solo está compuesta, en cambio, por propiedades específicas del lenguaje y específicas de la especie.

El componente medular de la FLE, afirman Chomsky y sus colegas, son los mecanismos computacionales de la "recursión" (para el PM, la operación de ensamble) que dan lugar a la propiedad de la "infinitud discreta", la capacidad de formar un número ilimitado de expresiones jerárquicamente estructuradas a partir de un conjunto finito de elementos. Junto con los mecanismos computacionales de la recursión, estos autores consideran que forma también parte de la facultad del lenguaje en sentido estricto lo que denominan "proyección a las interficies" (Hauser et al., 2002: 1573), esto es, "aspectos de la fonología, la semántica formal y el léxico en la medida en que satisfacen la condición de unicidad de la FLE" (Fitch et al., 2005: 182)9 . La tarea a la que se enfrentan los investigadores es, entonces, comparar el lenguaje humano con los sistemas de comunicación animal y con otros dominios cognitivos y determinar qué propiedades de la "proyección a las interficies" se integran bien en la FLE (son exclusivas tanto de la especie como del lenguaje), bien en la FLA (son compartidas por otras especies o están presentes en otros dominios cognitivos) ${ }^{10}$. Y no solo eso. Como se dice más arriba, la Tesis

${ }^{9}$ La distinción entre la FLA y la FLE resulta especialmente útil para valorar en sus justos términos el hallazgo del mal llamado "gen del lenguaje", el gen regulador FOXP2, cuya mutación es la causa del trastorno conocido como "disfasia genética" o "síndrome específico del lenguaje" (Specific Language Impairment). En palabras de Fitch et al. (2005: 190), "si hay algún candidato para ser incluido en la FLA, y no en la FLE, este es el gen FOXP2, un factor de transcripción fuertemente conservado que se encuentra en todos los mamíferos... y en todas las aves". Berwick y Chomsky (2011: 33-35) se extienden en este punto, e insisten en que "descubrimientos recientes en pájaros y ratones están conformando un creciente consenso en torno a la idea de que este gen no guarda relación con la sintaxis interna (la FLE), y menos aún es un hipotético gen del lenguaje (del mismo modo que no hay un único gen para el color de los ojos o el autismo), sino que forma parte de los mecanismos reguladores vinculados con la externalización". Precisan estos autores que "el FOXP2 participa en el desarrollo del control motórico serial fino, orofacial o de otro tipo: la habilidad, literalmente, de colocar un sonido o un gesto en el lugar apropiado y de manera sucesiva en el tiempo", y concluyen que "el FOXP2 no nos dice nada sobre el núcleo de la facultad del lenguaje humano, dado que en realidad no tiene que ver con el fenotipo de la facultad del lenguaje en sentido estricto (el ensamble y la sintaxis)". Sobre la disfasia genética y el gen FOXP2 véase, v.g., Longa (2006) y las referencias allí citadas.

${ }^{10}$ Véase la discusión en torno a esta cuestión entre Chomsky, Hauser y Fitch (2002), por un lado, y Steven Pinker y Ray Jackendoff (2005), por otro, en la que se exponen y analizan detallada- 
Minimista Fuerte nos obliga a comprobar también si la recursión (materializada por medio de la operación minimista de ensamble) pertenece a la facultad del lenguaje en sentido estricto y es, por tanto, específica del lenguaje y específica de la especie. Por razones de espacio, y dada su importancia, se revisará tan solo en lo que sigue algunas de las ideas que sobre esta última cuestión se han presentado recientemente.

Parece estar fuera de toda duda, después de décadas de intensas investigaciones sobre la comunicación animal, que la recursión, tal y como se manifiesta en el lenguaje humano, es una propiedad exclusiva de nuestra especie, que no se encuentra en ninguna otra especie animal, incluidos nuestros parientes más cercanos, y de la que tampoco ofrecen evidencias los experimentos con chimpancés, delfines o loros sometidos a entrenamiento (Fitch et al., 2005: 200; Jackendoff y Pinker, 2005: 217). Conviene matizar, no obstante, esta afirmación, ya que, como indican Hurford (2011) y Tallerman (2012), distintos estudios muestran que se puede observar algo semejante a la recursión en el canto de las aves y de otras especies, como las ballenas, en el que la combinación reglada de notas da lugar a unidades mayores con una limitada estructura jerárquica. Existe, sin embargo, una diferencia fundamental entre la sintaxis de las lenguas humanas y la sintaxis de los cantos de otras especies: como subrayan ambos autores, la sintaxis de las lenguas humanas es semánticamente composicional, de modo que el significado de una expresión es el resultado de la suma del significado de sus partes y de cómo se combinan. El canto de las aves y las ballenas no tiene, en cambio, una sintaxis semánticamente composicional, y se asemeja a los procesos fonológicos de las lenguas, en los que un conjunto finito de unidades sin significado (los fonemas) se combinan para formar unidades mayores (las sílabas), también carentes de significado. Chomsky, Hauser y Fitch llegan a la misma conclusión que Hurford y Tallerman en este punto: "solo los seres humanos tienen la capacidad de recombinar unidades dotadas de significado y formar con ellas una serie ilimitada de estructuras más complejas, obteniéndose en cada caso un significado distinto" (Hauser et al., 2002: 1576).

El panorama es bastante más complejo, sin embargo, cuando se compara la facultad del lenguaje con otras parcelas de la cognición humana. Hauser et al. (2002: 1571, 1578) dejan abierta la posibilidad de que la recursión haya surgido

mente los resultados de los estudios comparados sobre las propiedades fónicas, léxico-conceptuales y combinatorias del lenguaje humano y de los sistemas de comunicación animal (cf. Hauser et al., 2002; Fitch et al., 2005; Jackendoff y Pinker, 2005; Pinker y Jackendoff, 2005). Chomsky destaca a este respecto que ciertas propiedades de las unidades léxico-conceptuales de las lenguas humanas parecen ser específicas de la especie (Chomsky, 2005: 4; 2007: 7; 2010: 57-58; Berwick y Chomsky, 2011: 39; Hauser et al., 2002: 1576). El modo de comprobar que esto es así es realizar experimentos con animales como los descritos en Hauser (2009), en los que se trata de saber si determinadas especies no humanas tienen capacidades como la cuantificación numérica o la distinción singular-plural. Más difícil es precisar qué propiedades léxico-conceptuales son parte de la FLE y cuáles pertenecen, en realidad, al sistema del pensamiento de la FLA (cf., v.g., Fitch et al., 2005: 203). 
en la especie por razones ajenas al lenguaje e instan a que se investigue si esta propiedad está también presente en otros dominios cognitivos, como la cuantificación numérica, la navegación espacial o las relaciones sociales. Las propuestas que se han formulado a este respecto son de muy distinto tipo y dependen, en parte, de cuál sea la capacidad cognitiva que se analice.

En lo que atañe a la cuantificación numérica, por ejemplo, Chomsky (2005: 16; 2007: 5; 2008: 139; 2010: 53) sugiere que "la capacidad aritmética" podría derivarse de la recursión lingüística: la operación de ensamble, aplicada a un léxico con un solo miembro, da lugar al sistema de los números naturales. Jackendoff y Pinker (2005: 217-18) apuntan, por su parte, que el agrupamiento de unidades discretas para crear estructuras jerárquicas también es una característica de dominios cognitivos humanos como la visión o la música, pero señalan que hay propiedades formales que distinguen a la recursión sintáctica, entre ellas, el hecho de que los constituyentes sintácticos estén encabezados por un núcleo del que depende el resto de los miembros del conjunto. Dado que existen estructuras jerárquicas organizadas alrededor de un núcleo en otros dominios, como la estructura silábica o determinados aspectos de las estructuras musicales, Jackendoff y Pinker sostienen que la recursión sintáctica podría ser una combinación novedosa de propiedades que se encuentran en otras parcelas de la cognición.

Boeckx (2009) piensa igualmente que la estrategia de descomponer la recursión lingüística (la operación de ensamble) en operaciones más básicas puede ayudarnos a descubrir qué es lo que tiene de específico esta operación en comparación con otros sistemas recursivos, pero su propuesta no coincide con la de Jackendoff y Pinker. En opinión de este lingüista, en el ensamble participan dos procesos diferenciados: el "agrupamiento básico" (Basic Grouping), que produce estructuras jerárquicas, y la "operación de copia" (Copy), por la cual, cuando se combinan dos unidades lingüísticas, el conjunto resultante siempre tiene las propiedades de una de ellas (es endocéntrico). Para Boeckx, la especificidad de la recursión lingüística reside en el segundo de estos dos subcomponentes de la operación de ensamble. Noam Chomsky, en la sección de preguntas y comentarios que sigue al artículo de Boeckx, sostiene, en cambio, que la endocentricidad es un epifenómeno que se deriva de una condición de eficiencia computacional (la búsqueda mínima), y afirma que la propiedad que realmente distingue a la recursión lingüística es su carácter ilimitado (unboundedness).

De lo dicho hasta aquí se desprende que atribuir la operación de ensamble, y por ende cualquier otra propiedad, a la FLE (la GU, en términos de la lingüística chomskiana) no implica necesariamente elegir entre sí o no (o entre todo o nada). Se pueden concebir, como hemos visto, al menos otras tres opciones, que no se excluyen entre sí: a) el mecanismo de la recursión podría ser una propiedad prelingüística que se ha especializado en los seres humanos para desempeñar nuevas tareas (cf. Laka, 2009: 335), como la de combinar unidades con significado; b) 
lo específicamente lingüístico y humano tal vez sea algún subcomponente de la operación de ensamble; c) la instrucción genética para el lenguaje podría consistir en la combinación en una sola operación computacional de elementos compartidos con otros dominios cognitivos o con otras especies. Cualquiera de estas tres alternativas, o una mezcla de ellas, encajaría mejor con la idea de que el lenguaje surgió en época reciente y de manera abrupta (cf. \$3) que suponer que el ensamble lingüístico, con todas sus particularidades, quedó inscrito ex novo en el código genético de la especie.

Una última posibilidad, que reabre la vieja polémica sobre las relaciones entre lenguaje y pensamiento, es que la recursión sea una propiedad del sistema cognitivo lingüístico que se deriva de las condiciones de interficie (o arquitectura estructural) impuestas por el sistema conceptual-intencional. La idea, en palabras de Pinker y Jackendoff (2005: 230), sería que "la única razón por la que el lenguaje necesita ser recursivo es porque su función es expresar pensamientos recursivos". Un exponente reciente de esta idea, que hunde sus raíces en la concepción del lenguaje como expresión del pensamiento, es el libro de Michael C. Corballis, The recursive mind, en el que se postula, en esencia, que "los tipos de pensamiento que hicieron posible el lenguaje eran no lingüísticos, pero poseían, no obstante, propiedades recursivas a las que se adaptó el lenguaje" (Corballis, 2011: ix). Fundamenta Corballis esta aseveración en el análisis de dos tipos de pensamiento de naturaleza, a su entender, prelingüística y, probablemente, específicamente humana: el "viaje mental en el tiempo" (la habilidad de evocar sucesos del pasado e imaginar situaciones futuras) y lo que se conoce como "teoría de la mente" (poder adivinar lo que el otro está pensando). Ambas formas de pensamiento, nos recuerda este investigador, hacen uso de principios recursivos: en el primer caso, las situaciones pasadas o futuras pueden insertarse tanto en lo que en un momento dado se esté pensando como en otras situaciones evocadas o imaginadas; en el segundo, no solo es uno capaz de adivinar lo que el otro está pensando, sino que también puede uno saber que el otro sabe lo que uno está pensando. Para Corballis (2011), la recursión tiene, en definitiva, su origen en el pensamiento ${ }^{11}$.

El punto de vista opuesto se expone claramente en trabajos como los de Wol-

${ }^{11}$ Nótese que los casos estudiados por Corballis (2011) no ilustran exactamente el fenómeno de la recursión en el sentido que Hauser et al. (2002) y Fitch et al. (2005) dan a este término. Aunque no lo definen, de la lectura de estos textos se deduce que Chomsky y sus colegas entienden la recursión lingüística, en sentido amplio, como la capacidad de construir de manera ilimitada frases jerárquicamente estructuradas a partir de un número finito de unidades discretas. Corballis hace uso, en cambio, de este término, de manera más restringida, para nombrar la propiedad de la autoincrustación, un subtipo de estructura jerárquica en el que, en el caso del lenguaje, un constituyente se inserta dentro de otro constituyente de la misma categoría (v.g., el gato [que cazó el ratón [que se comió el queso $\left.]_{0}\right]_{0}$ ). Para evitar malentendidos, tal vez fuera conveniente usar el término "recursión" solo en su acepción más laxa ('construcción ilimitada de frases jerarquizadas') y emplear otro vocablo ("recursividad") para referirse al mecanismo, más específico, de la autoincrustación. 
fram Hinzen $(2006,2009,2011)$. Comparte este lingüista la idea, defendida desde antaño por autores de distintas tendencias, de que el lenguaje no expresa el pensamiento, sino que le da forma, y sostiene, en particular, que no existe pensamiento proposicional con independencia del lenguaje. En su opinión, las representaciones semánticas estructuradas se conforman, en concreto, como resultado de la aplicación de la operación sintáctica de ensamble a unidades con significado y de la creación por medio de procedimientos también gramaticales de la ontología universal del lenguaje, "el conjunto distintivo de categorías básicas en cuyos términos pensamos, como las de objeto, evento, estado de cosas o proposición, cada una de las cuales se corresponde con raíces léxicas insertas en determinadas configuraciones sintácticas" (Hinzen, 2011: 513) ${ }^{12}$. La conclusión a la que llega Hinzen (2009: 130) es, por tanto, que "no puede haber pensamientos [proposicionales] fuera de las formas posibles que proporciona el sistema computacional lingüístico". Desde esta perspectiva, al contrario de lo que piensa Corballis, el origen de la propiedad de la recursión está en el lenguaje, no en el pensamiento.

Llegando a este punto, lo relevante es saber si autores como Hinzen tienen o no razón; esto es, debe comprobarse si hay manifestaciones de pensamiento proposicional en especies no humanas o si alguno de nuestros predecesores en la familia de los homínidos podía pensar de manera proposicional sin lenguaje. Si finalmente se demostrara que se cumple uno u otro de estos supuestos (o ambos), la facultad del lenguaje en sentido estricto (la GU) aún debería incluir alguna propiedad específica de la especie y del lenguaje genéticamente establecida, aunque solo sea la adopción de la operación de ensamble para externalizar pensamientos recursivos o el modo particular en que se integran los distintos elementos que componen la facultad del lenguaje en sentido amplio ${ }^{13}$. Solo así se pueden explicar las diferencias que existen entre el lenguaje humano y los sistemas de comunicación de otras especies.

\section{EL MODELO DE ADQUISICIÓN DEL LENGUAJE}

La idea minimista de que la GU debe tener el menor contenido posible, para poder así explicar tanto las propiedades de la facultad del lenguaje como su evo-

\footnotetext{
${ }^{12}$ Véanse los detalles técnicos de esta idea en Hinzen (2011: 509 y ss.). La propuesta de Hinzen se aparta sustancialmente del modelo minimista de la facultad del lenguaje (cf. \$3), dado que, llevada hasta sus últimas consecuencias, implica que "no hay un componente semántico, un sistema del 'pensamiento' independiente del lenguaje, una 'proyección' desde la sintaxis a dicho sistema, una 'interficie’ semántica” (Hinzen, 2009: 128). Si Hinzen está en lo cierto, ninguna propiedad del sistema cognitivo lingüístico podría explicarse a partir de su interacción con el sistema del pensamiento. Se debilitaría así, en parte, la Tesis Minimista Fuerte y habría que suponer que la FLE (la GU) tiene más contenido del que el PM quisiera atribuirle.

${ }^{13}$ Cf. Chomsky (2004b: 162-163; 2007: 5; 2010: 53), Fitch et al. (2005: 181, 182, 201).
} 
lución (cf. \$3), obliga a revisar en profundidad el modelo de adquisición del lenguaje de la Teoría de los Principios y los Parámetros. Para la Teoría P\&P, en el desarrollo de las gramáticas mentales de los individuos intervienen dos factores: la dotación genética específica del lenguaje (la GU) y la experiencia lingüística. Según este modelo (cf., v.g., Chomsky, 1981, 1986), el niño que "aprende" su lengua materna dispone de antemano, en tanto que miembro de la especie, de un rico conocimiento lingüístico inscrito de algún modo en su código genético que incluye, entre otras cosas, un complejo sistema de principios invariables de buena formación y de parámetros de variación sintáctica, con opciones también predeterminadas. A partir de los datos lingüísticos que percibe en su entorno, el niño escoge una de estas opciones ("fija” los parámetros), produciéndose a menudo un efecto en cascada sobre parcelas de la gramática de las lenguas, y se obtiene como resultado un determinado sistema cognitivo lingüístico (o lengua-I). El modelo de adquisición del lenguaje de la Teoría P\&P, representado esquemáticamente en (9), es, por tanto, un modelo en el que la GU ocupa un lugar privilegiado, se trata de un modelo "con primacía de la GU”: en este modelo, en esencia, los estímulos ambientales (o datos lingüísticos primarios, DLP) activan la información contenida en la GU para seleccionar una gramática en particular (cf. Lorenzo y Longa, 2009).

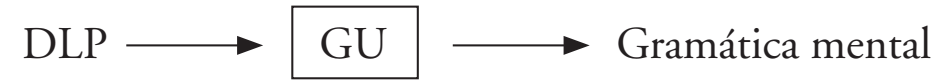

En el Programa Minimista, como veíamos con detalle en el apartado 3, se intenta reducir el contenido de la GU al máximo y se introduce en el modelo de adquisición del lenguaje un nuevo "tercer factor", formado por principios no específicos de la facultad del lenguaje, que intervienen, junto con la GU y la experiencia lingüística, en su desarrollo en la mente de los hablantes. Como ilustran las siguientes palabras de Chomsky, el centro de interés se desplaza en el PM de la GU a los mecanismos y principios del tercer factor, que desempeñarían ahora un papel central en el proceso de adquisición de una lengua:

En la historia moderna de la gramática generativa, el problema de la determinación del contenido de la facultad del lenguaje se ha abordado con una perspectiva "de arriba abajo": ¿cuánto contenido debe atribuirse a la GU para dar cuenta de la adquisición del lenguaje? El Programa Minimista pretende abordar este problema "de abajo arriba": ¿hasta qué punto puede reducirse el contenido de la GU a la vez que se sigue dando cuenta de la variedad de las lenguas-I que se adquieren, sobre la base de principios del tercer factor? (Chomsky, 2007: 4).

El esquema de (10), tomado de Lorenzo y Longa (2009), reproduce un modelo minimista de la adquisición del lenguaje con "primacía del tercer factor", en el 
que principios generales externos al lenguaje dan forma a gramáticas particulares, con la contribución de la experiencia lingüística y con una aportación mínima (idealmente nula) de la GU:

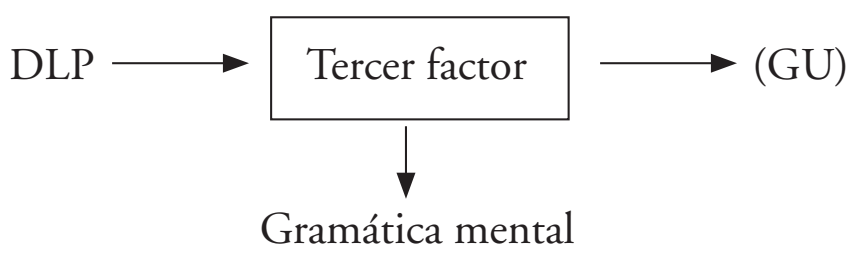

La inclusión del tercer factor en el modelo de adquisición del lenguaje implica que debe matizarse la tradicional caracterización de la GU como el estadio inicial $\left(\mathrm{E}_{0}\right)$ en el proceso de aprendizaje de la lengua materna, que restringe los tipos de gramáticas posibles (cf., v.g., Chomsky, 1965: 26-27; 1986: 52; 1995: 167, 169). También los principios del tercer factor forman parte de las precondiciones para el aprendizaje de una lengua (el "estadio inicial") y limitan los tipos posibles de lenguas. Debe distinguirse, así, como hacen Longa (2006) y Longa y Lorenzo (2008), entre rasgos innatos y rasgos genéticamente especificados. En palabras de Longa (2006: 202), “...un rasgo innato sería simplemente una propiedad determinada a aparecer de manera robusta y fiable en cierto punto del proceso de desarrollo de cualquier miembro de una especie, con independencia de los fundamentos de su desarrollo, que pueden ser genéticos, pero también de otros tipos”. De este modo, "una propiedad determinada de la gramática puede seguir siendo considerada innata, aunque no responda a una instrucción gramatical específica a partir de una especificación genética” (Longa, 2006: 203). Las condiciones de eficiencia computacional serían un ejemplo de propiedades innatas (pertenecientes a $\mathrm{E}_{0}$ ) que no están inscritas en el código genético. La operación de ensamble, por su parte, tal vez sea una propiedad innata genéticamente especificada (cf. \$4). A la vista de lo dicho, y para ser más precisos, la GU debería caracterizarse, por lo tanto, no como el estadio inicial para la adquisición del lenguaje, sino como el componente de $\mathrm{E}_{0}$ con un fundamento genético específico de la especie.

El hecho de que, en el PM, los principios del tercer factor conformen, junto con la GU, el estadio inicial de la adquisición del lenguaje nos lleva a relativizar, igualmente, el peso que en la Teoría P\&P tiene el argumento de la pobreza del estímulo en la justificación de una GU sobredimensionada (cf. \$2). Dicho argumento avala, ciertamente, la idea de que el conocimiento lingüístico de los hablantes está en parte predeterminado, pero no nos dice cómo. En el modelo minimista de adquisición, la brecha entre la pobreza de los estímulos lingüísticos del entorno y la riqueza del sistema cognitivo lingüístico que se desarrolla en la mente del niño se estrecha proponiendo que existen propiedades "innatas" tanto del primer factor (la GU) como del tercer factor. Así las cosas, la "pobreza del estímulo" pierde fuerza, a mi entender, como argumento no solo a favor de que la GU esté dotada de 
un rico contenido, sino en apoyo incluso de la existencia misma de la GU.

El modelo minimista de adquisición del lenguaje de (10) abre líneas de investigación novedosas dentro de la lingüística de corte chomskiano. La primera de ellas es identificar los mecanismos no específicos de la facultad del lenguaje pertenecientes al "tercer factor" que inciden en el aprendizaje del lenguaje y delimitar con precisión cuál es su papel en el proceso de adquisición de las lenguas maternas, prestando especial atención tanto a las condiciones de arquitectura estructural y computación eficiente que, para el PM, explican propiedades centrales del sistema cognitivo lingüístico (cf. Chomsky, 2005: 6), como a principios de análisis de datos y estrategias de aprendizaje que se aplican en otros dominios (cf. Chomsky, 2010: 61).

Un buen ejemplo de esta línea de investigación es el "modelo variacional" de la adquisición del lenguaje de Charles Yang (2002, 2010), que incorpora mecanismos generales de aprendizaje, en concreto, nociones estadísticas (efectos de frecuencia en la fijación de los parámetros), para dar cuenta de la gradualidad y la falta de uniformidad del lenguaje infantil. El modelo de Yang adopta, no obstante, la Teoría de los Principios y los Parámetros. Asume este autor, por tanto, que el "espacio de variación" está predeterminado por la GU, aunque se aparta de la visión estándar de la parametrización de la Teoría P\&P (cf. supra) y propone que lo que la GU define es un conjunto de gramáticas "accesibles para quien aprende una lengua desde el principio" (Yang, 2002: 26), que compiten para ajustarse a los datos lingüísticos del entorno.

Con independencia de cómo se materialice, la idea de que las opciones de variación sintáctica forman parte de la GU, que el modelo de Yang comparte, es incompatible, como sabemos (cf. \$3), con la infraespecificación minimista del contenido de la dotación genética para el lenguaje específica de la especie. Fieles a los postulados del PM, un número cada vez mayor de investigadores que trabajan dentro de este marco teórico sostiene, en consecuencia, que los parámetros no pertenecen a la GU (el primer factor), sino que son propiedades emergentes (epigenéticas) de los sistemas lingüísticos que surgen como resultado de la interacción de principios no específicos del lenguaje (el tercer factor) con la experiencia lingüística (el segundo factor). Como mencionaba en el apartado 3, un ejemplo paradigmático de esta nueva concepción de la naturaleza de los parámetros de variación sintáctica es la explicación que actualmente se da en el PM de los valores y las correlaciones gramaticales asociadas al Parámetro de la Posición del Núcleo: los valores de este parámetro (el núcleo precede o sigue a sus complementos) están impuestos por el sistema articulatorio-perceptivo, que exige que las unidades lingüísticas se sucedan en el tiempo, dando lugar a tan solo dos opciones; a este requisito no específico del lenguaje se añade un principio general del aprendizaje, perteneciente también al tercer factor, que favorece los sistemas armónicos (la "generalización del input"), y se recoge, así, el hecho de que los núcleos tienden 
a situarse delante o detrás de sus complementos de manera homogénea en las lenguas del mundo.

Habiendo reubicado la fuente de la parametrización en el segundo y el tercer factor, otra importante línea de investigación que el modelo minimista de adquisición del lenguaje inaugura, todo un reto en realidad, es explicar, recurriendo a condiciones de estos dos factores, cómo surgen los articulados y restrictivos patrones de variación sintáctica que sistemáticamente se observan cuando se comparan unas lenguas con otras, en particular, los agrupamientos de propiedades gramaticales vinculadas con determinados parámetros, como el Parámetro del Sujeto Nulo (cf. \$2), y las redes jerárquicas de parámetros interrelacionados (cf. Roberts y Holmberg, 2010).

\section{REFERENCIAS}

Berwick, Robert C. y Chomsky, Noam. (2011). The biolinguistic program: The current state of its development. En A. M. Di Sciullo y C. Boeckx (eds.), The biolinguistic enterprise: New perspectives on the evolution and nature of the human language faculty (pp. 19-41). Oxford: Oxford University Press.

Boeckx, Cedric. (2009). The nature of Merge: Consequences for language, mind and biology. En M. Piattelli-Palmarini, J. Uriagereka y P. Salaburu (eds.), On minds and language. A dialogue with Noam Chomsky in the Basque Country (pp. 44-57). Oxford: Oxford University Press.

Boeckx, Cedric. (2011). Approaching parameters from below. En A. M. Di Sciullo y C. Boeckx (eds.), The biolinguistic enterprise: New perspectives on the evolution and nature of the human language faculty (pp. 205-221). Oxford: Oxford University Press.

Chomsky, Noam. (1965). Aspects of the Theory of Syntax. Cambridge, MA: The MIT Press. [Trad. esp.: Aspectos de la teoría de la sintaxis. (1970). Madrid: Aguilar].

Chomsky, Noam. (1981). Lectures on government and binding. Dordrecht: Foris.

Chomsky, Noam. (1986). Knowledge of language: Its nature, Origin, and Use. Nueva York: Praeger.

Chomsky, Noam. (1995). The Minimalist Program. Cambridge, MA: The MIT Press.

Chomsky, Noam. (2000). Minimalist inquiries: The framework. En R. Martin et al. (eds.), Step by step. Essays on minimalist syntax in honor of Howard Lasnik (pp. 89-155). Cambridge, MA: The MIT Press.

Chomsky, Noam. (2001). Derivation by phase. En M. Kenstowicz (ed.), Ken Hale: A life in language (pp. 1-52). Cambridge, MA: The MIT Press.

Chomsky, Noam. (2004a). Beyond explanatory adequacy. En A. Belletti (ed.), 
Structures and beyond (pp. 104-131). Oxford: Oxford University Press.

Chomsky, Noam. (2004b). The generative enterprise revisited. Berlín: Mouton de Gruyter.

Chomsky, Noam. (2005). Three factors in language design. Linguistic Inquiry, 36.1, 1-22.

Chomsky, Noam. (2007). Approaching UG from below. En U. Sauerland y H.M. Gärtner (eds.), Interfaces+recursion = language? Chomsky's minimalism and the view from syntax-semantics (pp. 1-30). Nueva York: Mouton de Gruyter.

Chomsky, Noam. (2008). On phases. En C. P. Otero, R. Freidin y Ma L. Zubizarreta (eds.), Foundational issues in linguistics (pp. 133-166). Cambridge, MA: The MIT Press.

Chomsky, Noam. (2010). Some simple evo-devo theses: How true might they be for language? En R. K. Larson, V. Déprez y H. Yamakido (eds.), The evolution of human language: Biolinguistic perspectives (pp. 45-62). Cambridge: Cambridge University Press.

Chomsky, Noam y Lasnik, Howard. (1993). The theory of principles and parameters. En J. Jacobs et al. (eds.) Syntax: An international handbook of contemporary research, vol. 1 (pp. 506-569). Berlín: Walter de Gruyter.

Corballis, Michael C. (2011). The recursive mind. The origins of human language, thought, and civilization. Princeton: Princeton University Press.

Eguren, Luis y Fernández Soriano, Olga. (2004). Introducción a una sintaxis minimista. Madrid: Gredos.

Fitch, W. Tecumseh, Hauser, Marc D. y Chomsky, Noam. (2005). The evolution of the language faculty. Clarifications and implications. Cognition, 97, 179210.

Gould, Stephen Jay. (2002). The structure of evolutionary theory. Cambridge, MA: Belknap Press. [Trad. esp.: La estructura de la teoría de la evolución. (2004). Barcelona: Tusquets].

Hauser, Marc D. (2009). Evolingo. The nature of the language faculty. En M. Piattelli-Palmarini, J. Uriagereka y P. Salaburu (eds.), On minds and language. A dialogue with Noam Chomsky in the Basque Country (pp. 74-84). Oxford: Oxford University Press.

Hauser, Marc D.; Chomsky, Noam y Fitch, W. Tecumseh. (2002). The faculty of language: what is it, who has it and how did it evolve? Science, 298, 1569-1579.

Hinzen, Wolfram. (2006). Mind design and minimal syntax. Oxford: Oxford University Press.

Hinzen, Wolfram. (2009). Hierarchy, Merge, and truth. En M. Piattelli-Palmarini, J. Uriagereka y P. Salaburu (eds.), On minds and language. A dialogue with Noam Chomsky in the Basque Country (pp. 123-141). Oxford: Oxford University Press.

Hinzen, Wolfram. (2011). Language and thought. En C. Boeckx (ed.), The Oxford 
handbook of linguistic minimalism. (pp. 499-522). Oxford: Oxford University Press.

Holmberg, Anders. (2010). Parameters in minimalist theory: The case of Scandinavian. Theoretical Linguistics, 36.1, 1-48.

Hurford, James. (2011). The origins of grammar. Oxford: Oxford University Press. Jackendoff, Ray y Pinker, Steven. (2005). The nature of the language faculty and its implications for evolution of language. Cognition, 97, 211-225.

Laka, Itziar. (2009). What is there in Universal Grammar? On innate and specific aspects of language. En M. Piattelli-Palmarini, J. Uriagereka y P. Salaburu (eds.), On minds and language. A dialogue with Noam Chomsky in the Basque Country (pp. 329-343). Oxford: Oxford University Press.

Longa, Víctor. (2006). Sobre el significado del descubrimiento del gen FOXP2. ELUA, 20, 177-207.

Longa, Víctor M. y Lorenzo, Guillermo. (2008). What about a (really) minimalist theory of language acquisition? Linguistics, 46.3, 541-570.

Longa, Víctor, Lorenzo, Guillermo y Uriagereka, Juan. (2011). Minimizing language evolution: The minimalist program and the evolutionary shaping of language. En Cedric Boeckx (ed.), The Oxford handbook of linguistic minimalism (pp. 595-616). Oxford: Oxford University Press.

Lorenzo, Guillermo y Longa, Víctor. (2009). Beyond generative geneticism: Rethinking language acquisition from a developmentalist point of view. Lingua, 119.9, 1300-1315.

Monod, Jacques. (1970). Le hasard et la nécessité. París: Editions du Seuil. [Trad. esp.: El azar y la necesidad. (1981). Barcelona: Tusquets].

Pinker, Steven y Jackendoff, Ray. (2005). The faculty of language: What's special about it? Cognition, 95, 201-236.

Roberts, Ian y Holmberg, Anders. (2005). On the role of parameters in Universal Grammar: A reply to Newmeyer. En H. Broekhuis, N. Corver, M. Everaert y J. Koster (eds.), Organizing grammar: A Festschrift for Henk van Riemsdijk (pp. 538-553). Berlín: Mouton de Gruyter.

Roberts, Ian y Holmberg, Anders. (2010). Introduction: Parameters in minimalist theory. En T. Biberauer, A. Holmberg, I. Roberts y M. Sheehan (eds.), Parametric variation: Null subjects in minimalist theory (pp. 1-57). Cambridge: Cambridge University Press.

Tallerman, Maggie. (2012). What is syntax? En M. Tallerman y K. Gibson (eds.), The Oxford handbook of language evolution (pp. 441-455). Oxford: Oxford University Press.

Yang, Charles. (2002). Knowledge and learning in natural language. Oxford: Oxford University Press.

Yang, Charles. (2010). Three factors in language variation. Lingua, 120.5, 11601177. 